IDETC2019-97519

\title{
ASSESSMENT OF JOINT PARAMETERS IN A KINECT SENSOR BASED REHABILITATION GAME
}

\author{
Soumya K. Manna, Venketesh N. Dubey \\ Faculty of Science \& Technology \\ Bournemouth University, Poole, UK
}

\begin{abstract}
A Kinect sensor based basketball game is developed for delivering post-stroke exercises in association with a newly developed elbow exoskeleton. Few interesting features such as audio-visual feedback and scoring have been added to the game platform to enhance patient's engagement during exercises. After playing the game, the performance score has been calculated based on their reachable points and reaching time to measure their current health conditions. During exercises, joint parameters are measured using the motion capture technique of Kinect sensor. The measurement accuracy of Kinect sensor is validated by two comparative studies where two healthy subjects were asked to move elbow joint in front of Kinect sensor wearing the developed elbow exoskeleton. In the first study, the joint information collected from Kinect sensor was compared with the exoskeleton based sensor. In the next study, the length of upperarm and forearm measured by Kinect were compared with the standard anthropometric data. The measurement errors between Kinect and exoskeleton are turned out to be in the acceptable range; $1 \%$ for subject 1 and $0.44 \%$ for subject 2 in case of joint angle; $5.55 \%$ and $3.58 \%$ for subject 1 and subject 2 respectively in case of joint torque. The average errors of Kinect measurement as compared to the anthropometric data of the two subjects are $16.52 \%$ for upperarm length and $9.87 \%$ for forearm length. It shows that Kinect sensor can measure the activity of joint movement with a minimum margin of error.
\end{abstract}

Keywords: Rehabilitation, Kinect, Joint parameters, Unity, Measurement accuracy, Exoskeleton

\section{INTRODUCTION}

Post-stroke patients suffer from different neuro-muscular deficits which include problems in grasping, reaching, balancing, keeping the arm in a certain position or orientation [1]. To restore the functionality, neuro-motor skills of human can be improved by self-training. It has been proved that taskbased training can improve hamstring strength and spastic reflexes for chronic stroke patients [2]. The main aim of poststroke rehabilitation is to increase the percentage of patient's participation in rehabilitation task. If the amount of patient's participation during exercise gets higher, the recovery rate will be better. Majority of post-stroke patients are usually in old age group $(\geq 60)$ [3]. It has been found that patients normally loose interest after performing monotonous therapy for a long time [4]. Therefore, the modern advancement in technology has improved the post-stroke rehabilitation process with VR based exercise module [5], [6]. It has transformed the post-stroke exercises into more engaging and motivating through gamebased therapy [7], therefore, these exercises become more enjoyable and clinically useful for patients.

On the other hand, patient's recovery condition should be determined by measuring different joint parameters after poststroke rehabilitation. Physical sensors such as EMG, IMU and accelerometers can be used to measure joint torque, inertia, angle, acceleration and so on. However, the mechanical look and complicated data acquisition system of these sensors have diminished the interest for using these sensors among patients group [8]. It is necessary to find an alternative way of measuring joint parameters accurately with less effort.

In fact, patients are now inclined towards an easy, comfortable and home-based rehabilitation system [9] rather than an expensive and highly sophisticated system used in rehabilitation centre. In order to overcome the limitations of physical sensors and to implement VR based rehabilitation therapy, few contactless motion capture systems have been developed such as Vicon [10], 3DMA [11], Kinect [12] etc. Due to the cost, accessibility, easy interfacing and user-friendly control, Kinect sensor based rehabilitation set-up has become a promising approach for post-stroke therapy. It can provide two advantages; new type of interesting and simple games suitable for post-stroke patients can create more interest among them to drive them back to exercises; also, the useful joint parameters and rehabilitation information can be extracted from Kinect sensor without using any contact sensor. These parameters can be used to evaluate patient's recovery rate. So far different types of rehabilitation game have been developed based on Kinect sensor such as bubble game [13], object catching game [14], table tennis [15] etc. The efficiency of these games is evaluated by three features; adaptation of post-stroke exercises through game oriented joint movements, performance evaluation after joint movement for clinical analysis, addition of more 
intriguing and user-friendly features for increasing user's interaction and participation in the game.

Over the last decade, Kinect sensor based game module has been upgraded and advanced to meet the desired objectives. As an example, task-based performance report is generated for patients after playing the game [8], illusion is created to motivate them for putting more effort [16], warning is given to patients if they follow the wrong track [17]. Also, mirror feedback is effective to improve the user's performance [18]. Patient's recovery status after exercise can be measured in two ways; performance report of the user in the game, analysis of measured kinematic and dynamic joint parameters. Each game module has its own performance criteria based on the number of attempts to achieve a goal [8] or the completion time [13]. In few games, different joint parameters such as position [19] and angle [20] and angular velocity [21] of the user are measured.

These measured joint parameters from Kinect sensor are not only used for accessing the recovery status of patients but also used as feedback signal in robotic devices for controlling the rehabilitation mode. For an example, trajectory tracking control has been implemented for robot added rehabilitation based on the joint parameters measured by Kinect sensor [22]. Kinect based skeleton data and measured joint parameters from EMG are fused together to monitor the rehabilitation process [23]. Research experiment has been performed in medical hospital to measure gait abnormalities using Kinect sensor [24]. Therefore, it is necessary to evaluate the measurement accuracy of Kinect sensor. Kinect based joint data is also compared with other motion capture technique such as $3 \mathrm{D}$ motion system [25]. The main aim of this paper is focused in two areas; i) enrichment of game based activities through scoring technique and audiovisual feedback while preserving the nature of rehabilitation activities ii) validation of Kinect measurement against other standard measurement approaches.

\section{METHODS}

A recently developed elbow exoskeleton [26] is used for the experiment. It is based on a hardware based mechanism and the whole operating regime of the exoskeleton has three subregions where each region comprises a specific actuation system for delivering particular rehabilitation mode in poststroke stages: Electric motor controlled exercise in the acute phase, assistance to patients through variable spring force in the mid-phase (Fig. 1) and variable resistive force in the last phase. In the first region, joint movements of user are controlled by electric motor however, in the next two regions users are free to initiate their own movements. In this research, the exoskeleton has been operated in the second region where users can bend their elbow with assistance from the exoskeleton. The exoskeleton has a potentiometer for measuring joint angle during motion. The internal values between adjacent joint angles are estimated by linear interpolation and these values have been passed through a smooth filter before plotting. Other joint parameters such as angular velocity and acceleration are measured by differentiating the joint angle with respect to time. Instead of using a rotary torque sensor, a mathematical model of joint torque has been developed in which joint parameters such as angle, velocity and acceleration are collected from the measured value and mass of the forearm supporting structure is computed from standard anthropometric data [27].
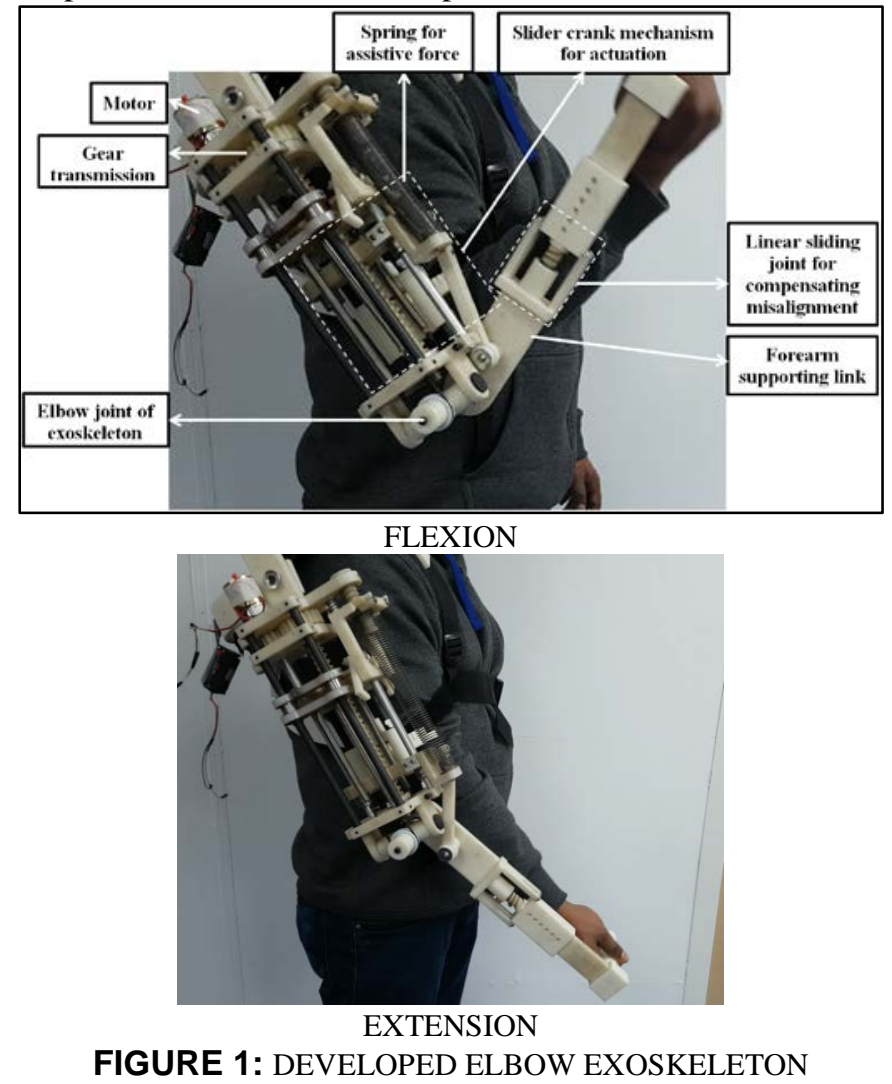

The basketball game (Fig. 2) has been developed [28] in unity game-engine where users can move the ball from the rest position to basket using arm gestures. The ball movement is controlled using the body tracking technique of Kinect sensor V2. After reaching the scoring position, user can basket the ball using a voice command 'drop'. Few user-friendly features are also added to the game to motivate patients such as scoring points, audio-visual feedback from the game environment and an avatar whose movement is same as the user for giving a real feel of exercise. The game is scripted in Microsoft visual studio.

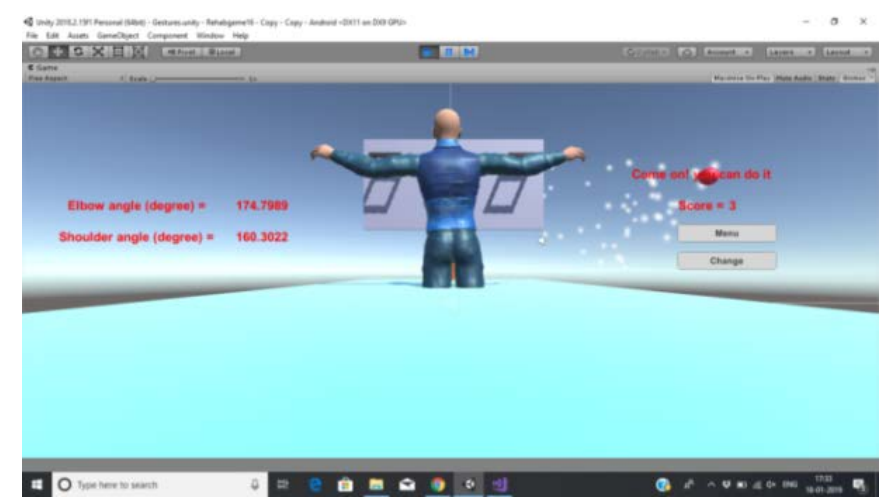




\section{FIGURE 2: DEVELOPED BASKETBALL GAME INTERFACE}

In this basketball game, users can utilize their elbow flexion-extension and shoulder abduction-adduction motion to control the ball. At the rest position, the joint angle of shoulder and elbow are $180^{\circ}$ whereas, at the scoring position, the joint angle of elbow and shoulder should be less than $90^{\circ}$ and $120^{\circ}$ respectively. The range of shoulder and elbow joint angle is divided into three stages (Fig. 3) to organize the difficulty of the game at three levels. Audio-visual feedbacks containing some motivating words are prompted and performance score will appear on screen after crossing each level. The working principle of the game is shown in flow chart Fig. 4. For evaluating the recovery status of users, joint vectors of human arm are measured using Kinect sensor and stored in an Excel file, therefore more joint features such as velocity, acceleration and torque are computed from the stored data using Matlab. Gravity force is applied to each game object. Windows speech library is used for voice reorganization. The holding contact between human hand and ball becomes zero when users utter the voice command 'drop', the ball would fall under gravity.

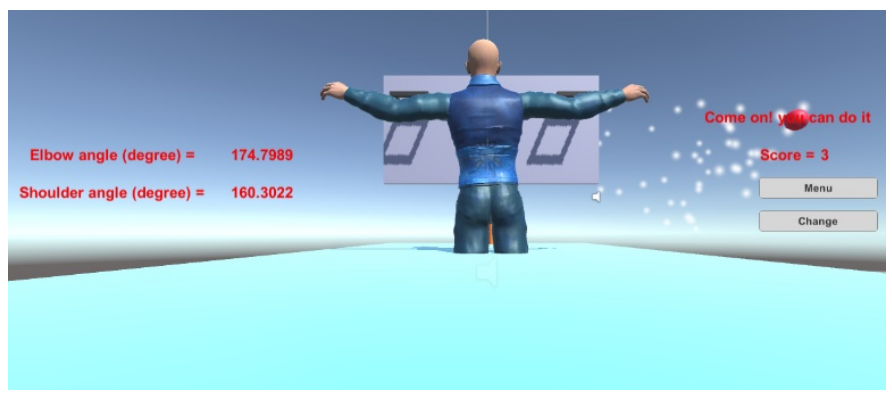

STAGE $1\left(\right.$ SHOULDER ANGLE $=180^{\circ}$, ELBOW ANGLE $\left.=180^{\circ}\right)$

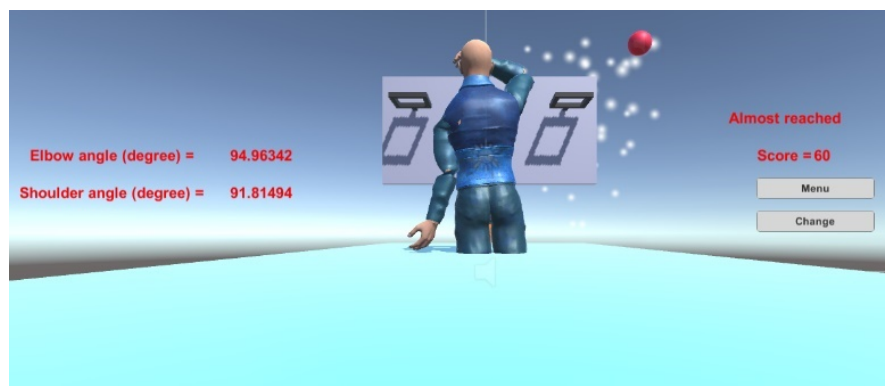

STAGE $2\left(\right.$ SHOULDER ANGLE $<150^{\circ}$, ELBOW ANGLE $<100^{\circ}$ )

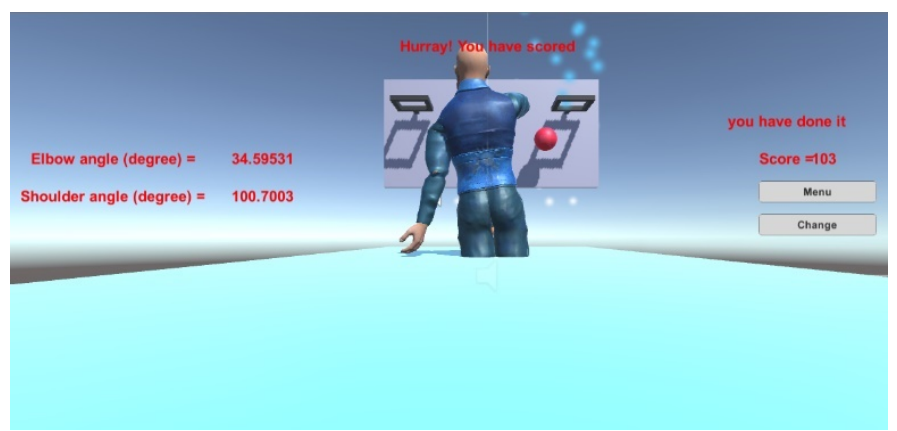

STAGE 3 (SHOULDER ANGLE $<120^{\circ}$, ELBOW ANGLE $<90^{\circ}$ )

FIGURE 3: THREE LEVELS OF THE GAME

Kinect sensor setup with personal computer

Modelling of the game in unity using 3D and 2D objects from unity scene and free graphics from unity asset store

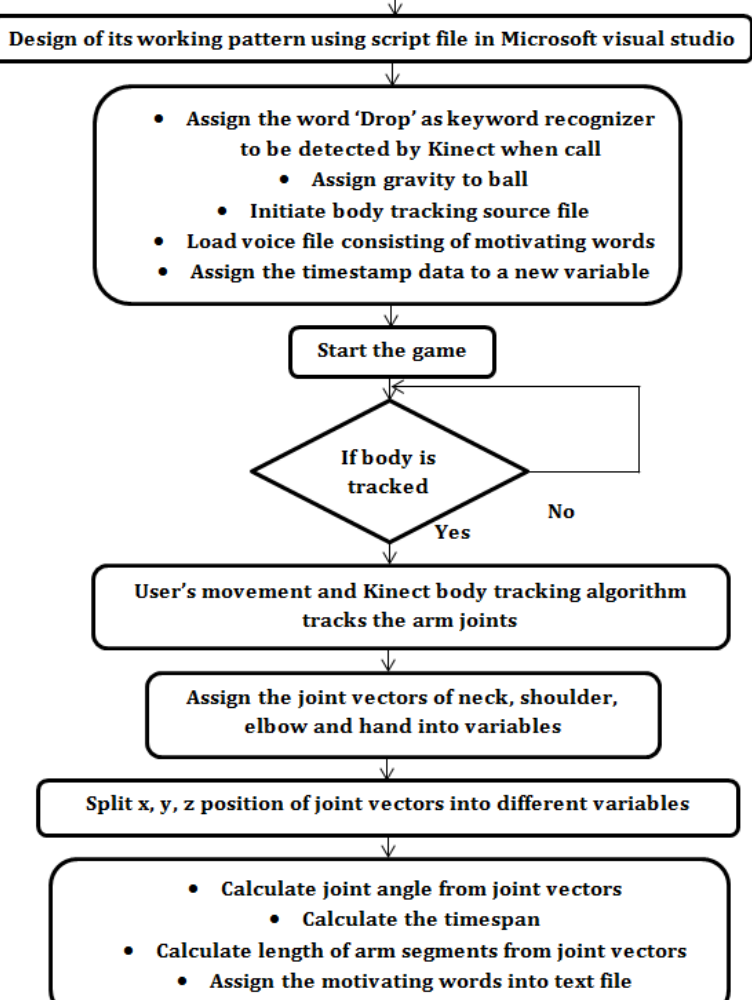

- Assign the word 'Drop' as keyword recognizer be detected by Kinect when call tracks the arm joint

the joint vecto ow and hand into variables 


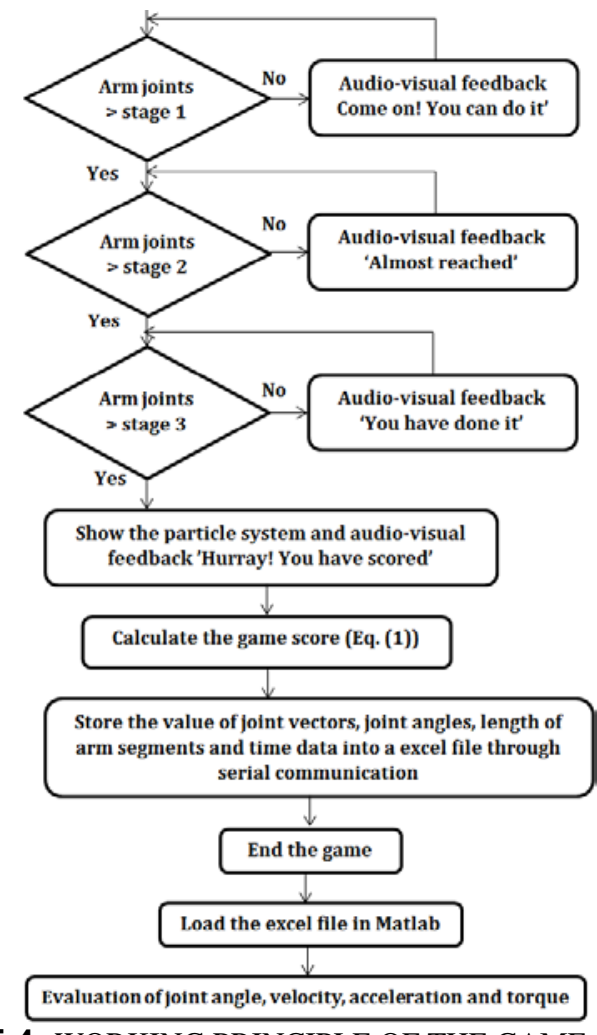

FIGURE 4: WORKING PRINCIPLE OF THE GAME

Two healthy subjects played the game in two situations. In the first case, they played the basketball game freely in front of Kinect sensor. The Kinect sensor captured the joint vectors of human arm by its inbuilt motion capture technique therefore joint angles and arm lengths are measured from the recorded data [28]. At the end of the game, the performance score is calculated based on user's maximum reachable joint angle and reaching time. The measured arm lengths from Kinect sensor are compared with calculated arm lengths from standard anthropometric data.

In the second case, these subjects moved their elbow in front of Kinect sensor wearing the developed exoskeleton. Physical sensors attached to the exoskeleton are used for measuring joint parameters. All these joint parameters are also measured through Kinect sensor. Joint torque of the user is calculated from the segment mass of the user, distance of centre of gravity and joint angle. If the measured joint parameters from exoskeleton are considered as standard, a comparative study is drawn between the sensor data of exoskeleton and Kinect measurement. The Kinect sensor is kept around 1.5 meter from the subject and is placed at 1.05 meter from the ground level. Kinect data based sensor data are passed through the low filter to remove the noise and the mean of consecutive five samples are taken.

\subsection{Scoring the game}

To measure the performance index of the user, the score after playing the game has been calculated based on their attained joint angle and reaching time. As users cross the boundary of upper stage, the shoulder angle and elbow angle is decreased. Also, minimum reaching time to the final scoring stage shows better performance. Therefore the formulation of the scoring technique is to create a higher score for better performance.

$$
=\frac{\left(\begin{array}{c}
\text { Score } \\
(180-\text { Maximum shoulder angle }(\text { degree }))+
\end{array}\right)}{\text { Reaching time }}
$$

\section{RESULTS AND DISCUSSION}

\subsection{Measured joint parameters of healthy subjects}

Users moved their right arm in front of a PC and Kinect sensor to play the game. Anthropometric data of these subjects are shown in Table 1 . Three stages of joint movement are shown in Fig. 5 for both subjects. The technique of measuring the joint parameters from joint vectors is shown in Fig. 6.

TABLE 1: ANTHROPOMETRIC DATA OF SUBJECTS

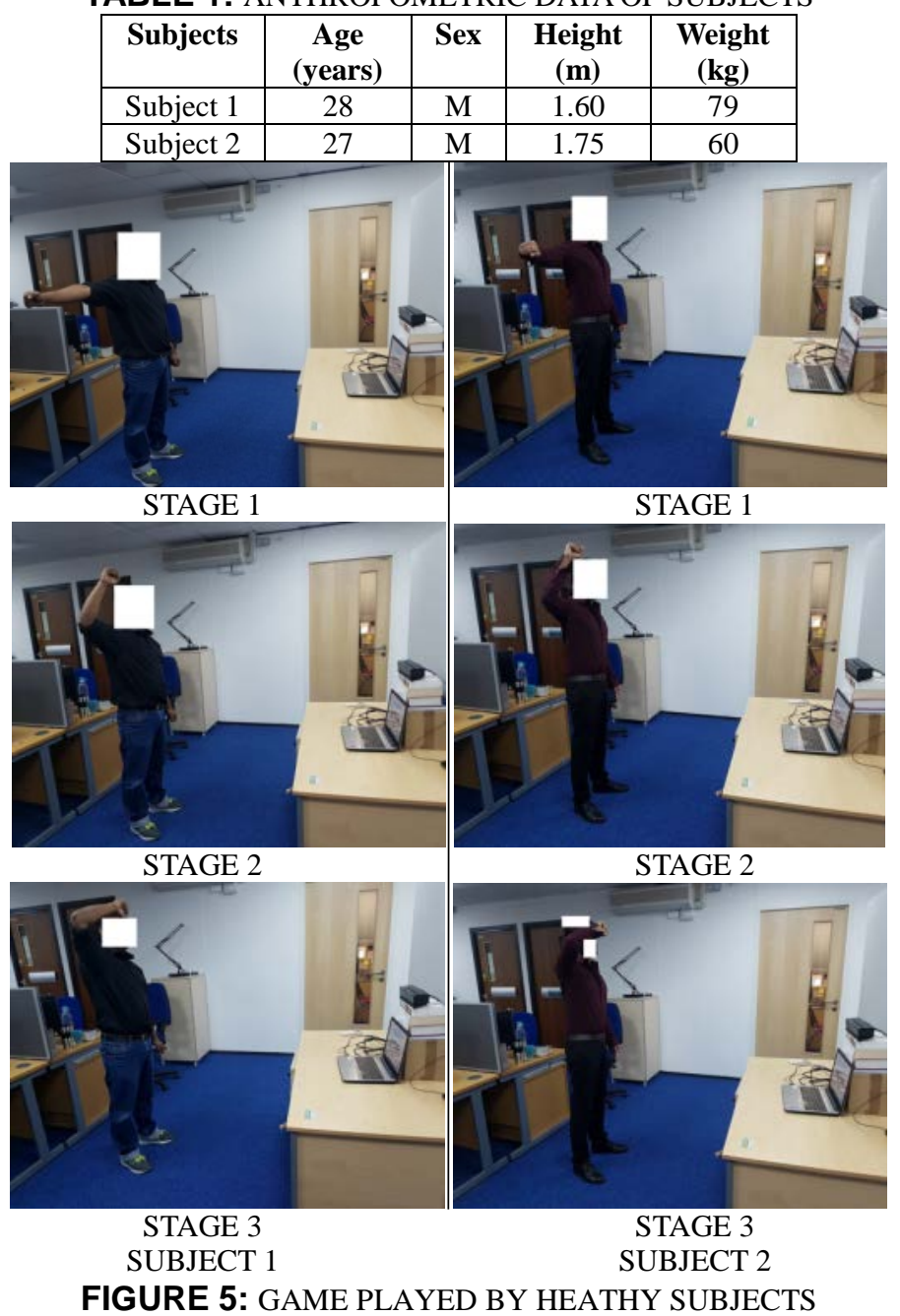




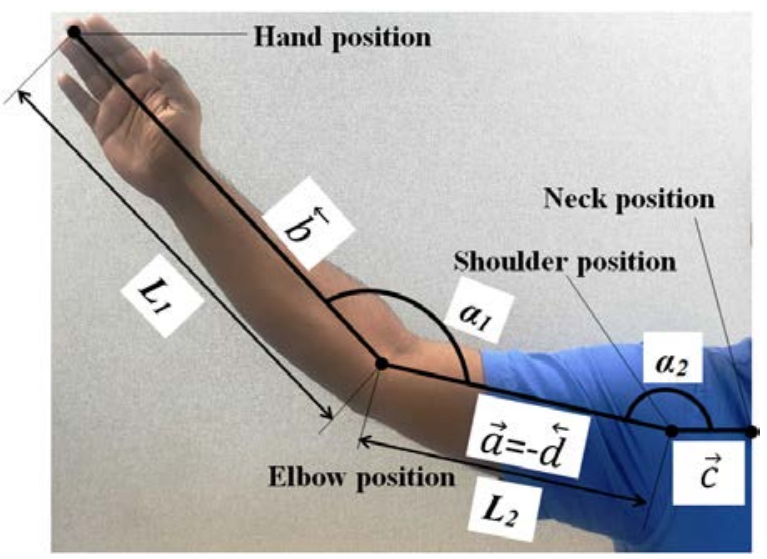

FIGURE 6: COMPUTATION OF JOINT PARAMETERS

Kinect SDK body tracking interface measures the position vector of neck, shoulder joint, elbow joint and distal end of hand (Fig. 6). The built-in body tracking algorithm of Kinect sensor can provide the position data with respect to the position of Kinect sensor as reference.

\section{As shown in Fig. 6,}

Length of upperarm $\left(L_{2}\right)=$ Length between the joint vectors of shoulder and elbow $=|\vec{a}|$

Length of forearm $\left(L_{1}\right)=$ Length between the joint vectors of elbow and hand $=|\vec{b}|$

Elbow joint angle $\left(\alpha_{1}\right)=\tan ^{-1} \frac{|a \times b|}{a \cdot b}$

Shoulder joint angle $\left(\alpha_{2}\right)=\tan ^{-1} \frac{|c \times d|}{c . d}$

$$
\text { Where } \vec{a}=-\overleftarrow{d}
$$

The covered joint angle of shoulder and elbow are shown in Fig. 7.

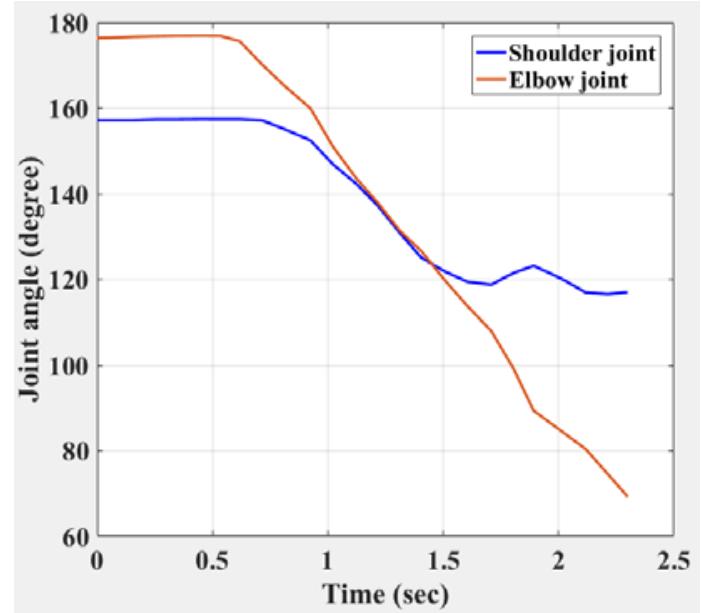

SUBJECT 1

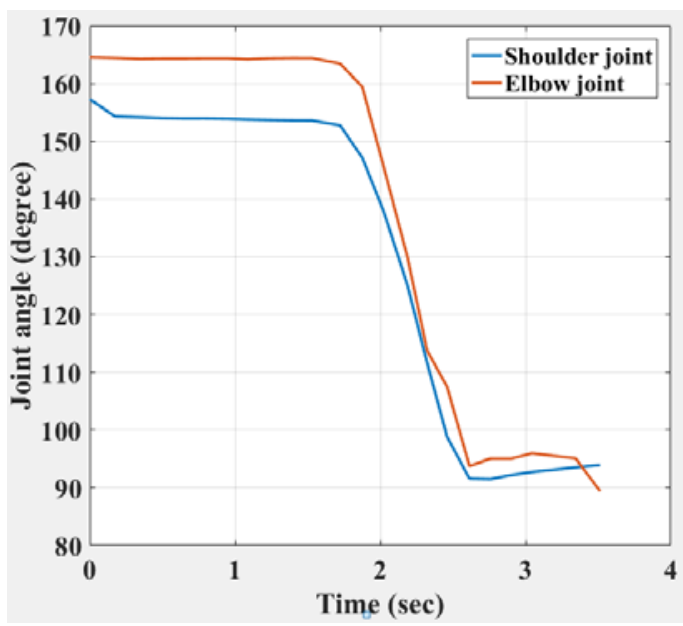

SUBJECT 2

FIGURE 7: JOINTS ANGLE MEASUREMENT USING KINECT

\subsection{Calculation of performance score}

As shown in Fig. 7, the maximum shoulder and elbow joint angle is $116.98^{\circ}$ and $69.29^{\circ}$ for subject $1 ; 93.98^{\circ}$ and $89.39^{\circ}$ for subject 2. As per the time frame, subject 1 took $2.29 \mathrm{sec}$ to reach the target whereas subject 2 took 3.512 sec to reach. As per Eq. (1), the performance score of both subjects are

$$
\begin{aligned}
& \text { Score }(\text { Subject } 1)=\frac{((180-116.98)+(180-69.29))}{2.29}=75.86 \\
& \text { Score }(\text { Subject } 2)=\frac{((180-93.98)+(180-89.39))}{3.51}=50.29
\end{aligned}
$$

Though both subjects have reached the target, it shows that the first subject has performed well in the game in terms of reach and time.

\subsection{Comparison of arm lengths with standard anthropometric data}

The standard arm segment length can be calculated as per the standard biomechanics rule [27],

Length of upper arm $=0.186 \times H$

Length of forearm $=0.254 \times H$

Where $H=$ Height of the user

Table 2 shows the standard length of arm segments of two subjects measured from anthropometric data and Kinect sensor.

TABLE 2: LENGTH OF ARM SEGMENTS (KINECT AND STANDARD ANTHROPOMETRIC DATA)

\begin{tabular}{|l|c|c|c|c|}
\hline Subjects & \multicolumn{2}{|c|}{ Anthropometric data } & \multicolumn{2}{c|}{ Kinect measurement } \\
\cline { 2 - 5 } & $\begin{array}{c}\text { Upperarm } \\
\text { length (m) }\end{array}$ & $\begin{array}{c}\text { Forearm } \\
\text { length (m) }\end{array}$ & $\begin{array}{c}\text { Upperarm } \\
\text { length (m) }\end{array}$ & $\begin{array}{c}\text { Forearm } \\
\text { length (m) }\end{array}$ \\
\hline Subject 1 & 0.29 & 0.41 & .24 & 0.37 \\
\hline Subject 2 & 0.33 & 0.45 & .28 & 0.40 \\
\hline
\end{tabular}


As the standard anthropometric data is considered to be the reference, therefore the percentage of error in Kinect measurement is calculated as

Error $(\%)=\frac{L(\text { Anthropometric })-L(\text { Kinect })}{L(\text { Anthropometric })} \times 100$

The average errors of Kinect measurement compared to the anthropometric data of two healthy subjects are $16.52 \%$ for upperarm length and $9.87 \%$ for forearm length, as shown in Table 3.

TABLE 3: ERROR IN THE MEASUREMENT OF ARM SEGMENTS

\begin{tabular}{|c|c|c|}
\hline Subjects & $\begin{array}{c}\text { Error in the } \\
\text { measurement in } \\
\text { upper arm length }\end{array}$ & $\begin{array}{c}\text { Error in the } \\
\text { measurement in } \\
\text { forearm length }\end{array}$ \\
\hline Subject 1 & $19.19 \%$ & $9.85 \%$ \\
\hline Subject 2 & $13.84 \%$ & $9.89 \%$ \\
\hline Average error & $16.52 \%$ & $9.87 \%$ \\
\hline
\end{tabular}

\subsection{Comparison of joint parameters with the elbow exoskeleton}

In this case, subjects can only move the basketball using elbow gestures when they are allowed to initiate free movements wearing the exoskeleton. As the first rehabilitation mode of the exoskeleton is controlled by motor, the subjects used the assistive mode of exoskeleton for elbow rotation (Fig. 8). All three joint parameters of elbow: angle, velocity and acceleration are measured using the sensor attached to the exoskeleton. For measuring dynamic elbow joint torque, movement of forearm is considered to be of one DOF manipulator, therefore, joint angle, velocity and acceleration are included in the calculation. The amount of assistive force and joint stiffness of the exoskeleton have been ignored in this assessment. On the other hand, these joint parameters are also measured by Kinect sensor using the developed motion capturing method. A comparative study is described between the sensor data of exoskeleton and Kinect sensor to evaluate the accuracy of Kinect measurement. The joint measurement error is calculated for joint angles and torque of both subjects (Fig. 9 to Fig. 12).

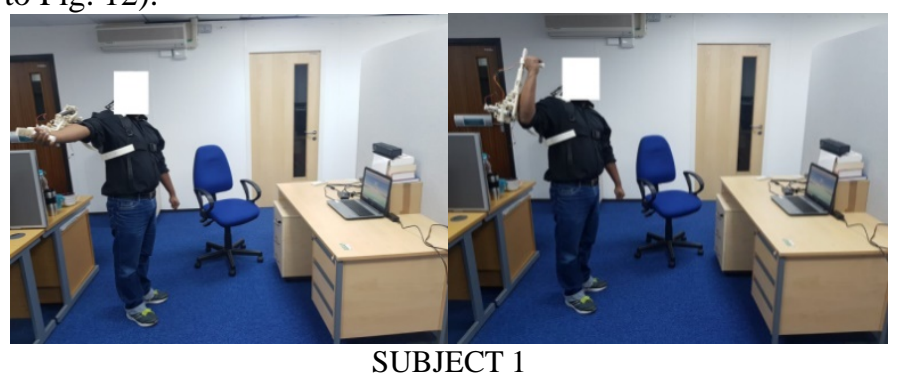

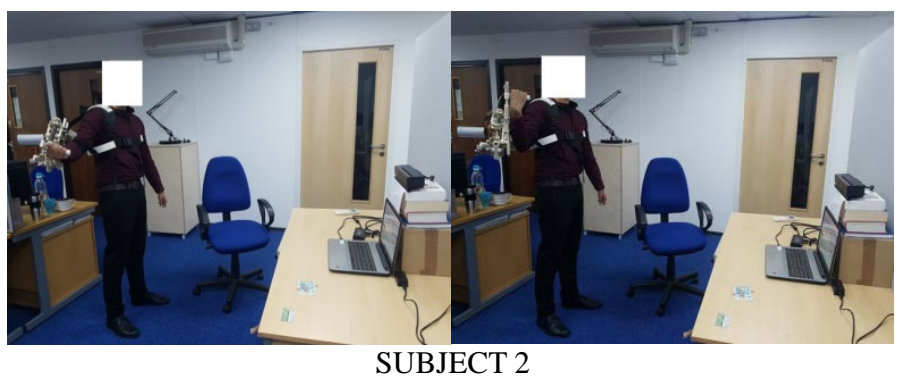

FIGURE 8: ELBOW ROTATION OF USER WEARING EXOSKELETON IN FRONT OF KINECT

As the measured joint parameters from exoskeleton are considered as the reference, measurement error in Kinect is calculated in Eq. (5).

Error $(\%)=\frac{L(\text { Exoskeleton })-L(\text { Kinect })}{L(\text { Exoskeleton })} \times 100$

The mean error for joint angle is small for both subjects; $1.076 \%$ for subject 1 and $0.44 \%$ for subject 2 .
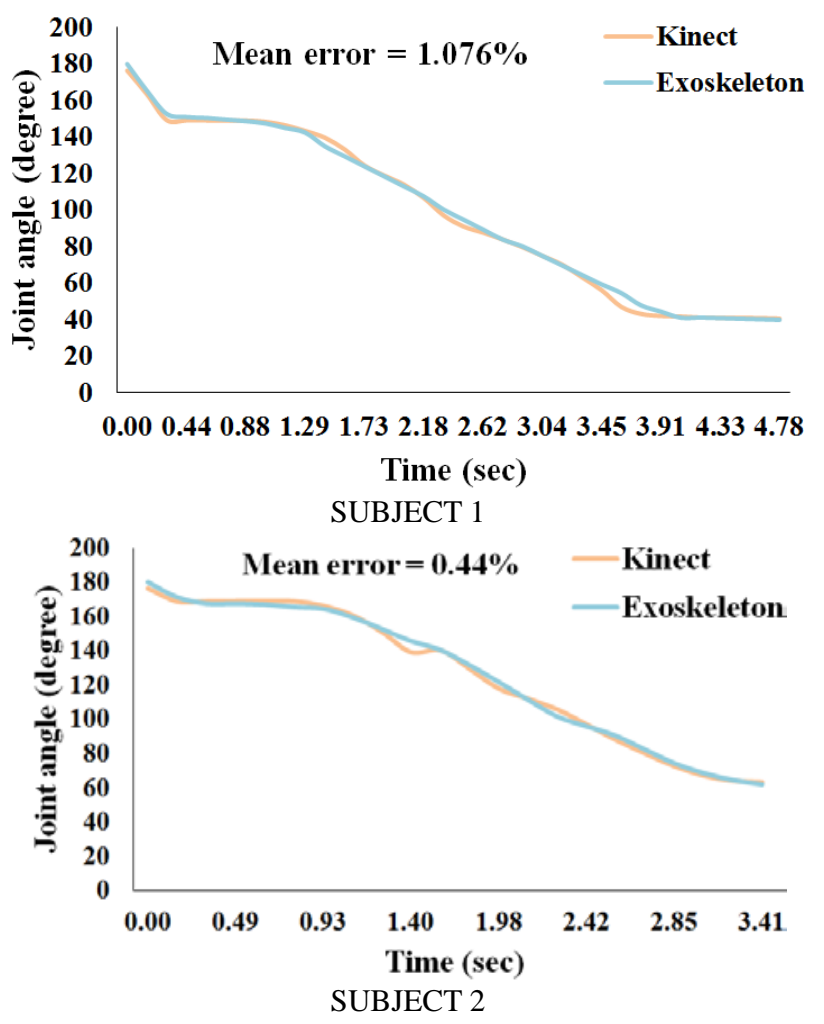

FIGURE 9: COMPARISON OF JOINT ANGLE MEASURED BY KINECT AND EXOSKELETON 

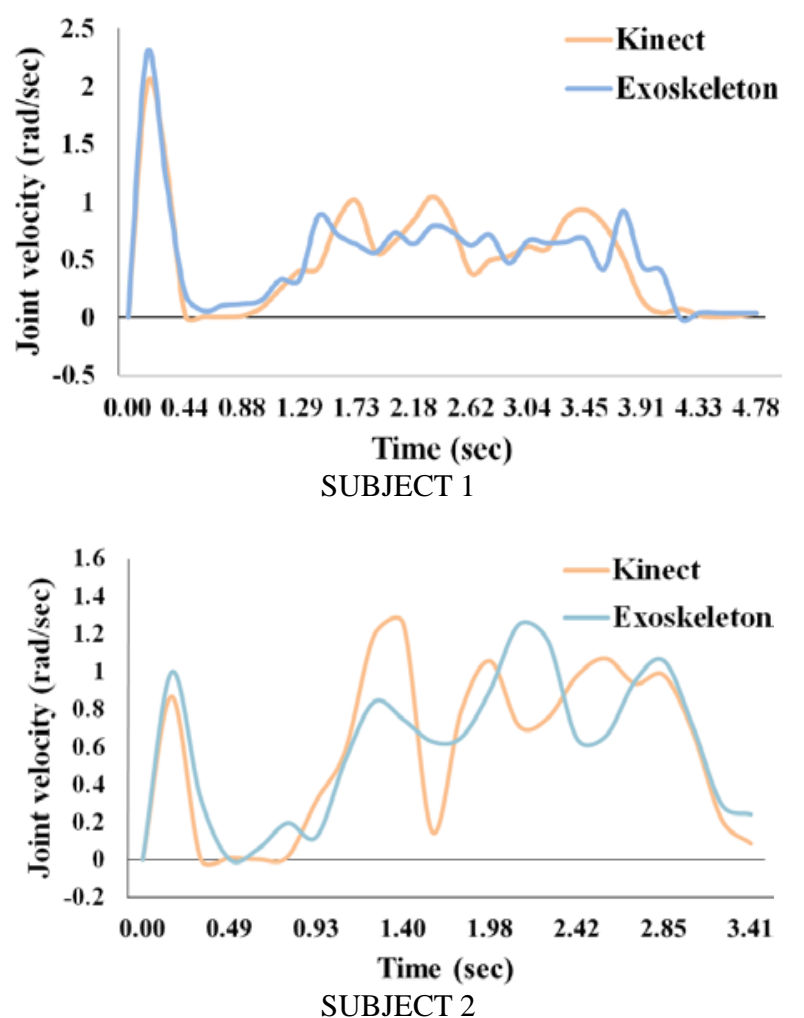

FIGURE 10: COMPARISON OF JOINT VELOCITY MEASURED BY KINECT AND EXOSKELETON
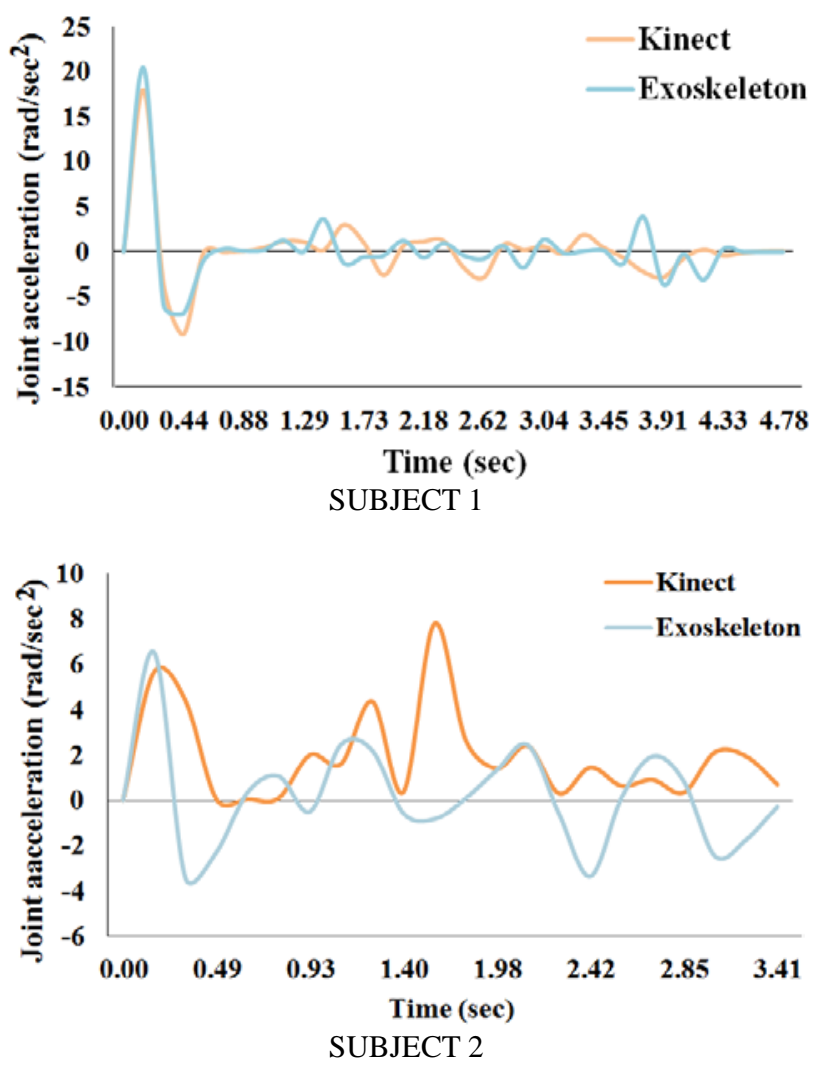

FIGURE 11: COMPARISON OF JOINT ACCELERATION MEASURED BY KINECT AND EXOSKELETON

\section{- Joint torque measurement}

For measuring the centre of gravity and the mass of forearm, the ratio between the arm segment and total body is considered as per the biomechanics rule [27].

The length between elbow joint and centre of mass of forearm $\left(l_{1}\right)=0.682 \times$ Forearm length

Weight of forearm supporting structure $\left(w_{1}\right)$

$=$ Weight of forearm + Weight of the forearm supporting structure of exoskeleton

$=0.022 \times W+W_{1}$

$W=$ User's body weight $(\mathrm{kg})$

$W_{1}=0.2 \mathrm{~kg}$

The inertia of forearm supporting structure with respect to the centre of mass is $I_{1}=w_{1} \times\left(L_{1} \times 0.468\right)^{2} \mathrm{~kg} . \mathrm{m}^{2}$

where $\left(L_{1} \times 0.468\right)=$ Radius of gyration with respect to the center of mass

Mass of forearm is same for both measurements: exoskeleton and Kinect whereas the values of forearm length and joint parameters are different. In case of exoskeleton, the forearm length is taken from the anthropometric data whereas Kinect sensor calculates the forearm length from the recorded joint vectors of human arm as shown in Table 2. The arm parameters for both measurements are shown in Table 4.

TABLE 4: COMPARISON OF ARM PARAMETERS OF TWO HEALTHY SUBJECTS

\begin{tabular}{|c|c|c|c|c|}
\hline Method & Subjects & $\begin{array}{c}\text { Forearm } \\
\text { weight } \\
\text { (kg) }\end{array}$ & $\begin{array}{c}\text { Length of } \\
\text { centre of } \\
\text { mass from } \\
\text { proximal } \\
\text { end (m) }\end{array}$ & $\begin{array}{c}\text { Radius of } \\
\text { gyration } \\
\text { with } \\
\text { respect to } \\
\text { the } \\
\text { centre of } \\
\text { mass (m) }\end{array}$ \\
\hline Exoskeleton & Subject 1 & 1.738 & 0.28 & 0.19 \\
\cline { 2 - 5 } & Subject 2 & 1.32 & 0.30 & 0.21 \\
\hline Kinect & Subject 1 & 1.738 & 0.25 & 0.17 \\
\cline { 2 - 5 } & Subject 2 & 1.32 & 0.273 & 0.19 \\
\hline
\end{tabular}

As per the Euler-Lagrange formulation of one DOF manipulator, joint torque of elbow is

$\tau_{\text {elbow }}=\left[w_{1} l_{1}^{2}+I_{1}\right] \ddot{\alpha}_{1}+w_{1} g l_{1} \cos \alpha_{1}$

Where $\alpha_{1}=\left(180-\theta_{1}\right)$

and $\theta_{1}=$ Elbow angle

The comparison of joint torque from both measurements is shown in Fig. 12. The mean error for joint torque is not very high for both subjects: $5.55 \%$ for subject 1 and $3.58 \%$ for subject 2 . 


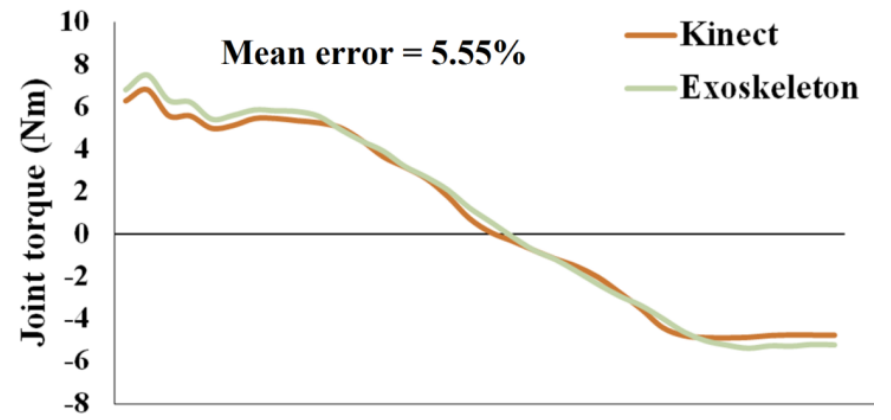

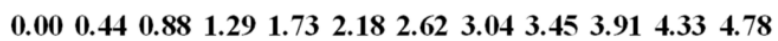

Time (sec)

SUBJECT 1

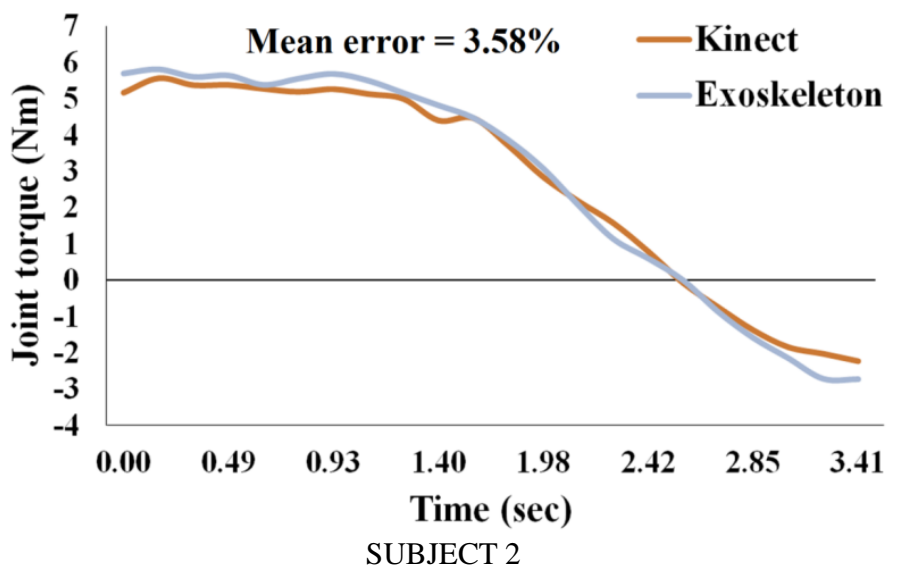

FIGURE 12: COMPARISON OF JOINT TORQUE MEASURED BY KINECT AND EXOSKELETON

\section{CONCLUSION}

To enhance user's participation in exercises, a Kinect sensor based voice controlled basketball game was developed since it engaged patients with fun based activities during the rehabilitation process. It is only possible when users have some residual muscle strength to undertake exercises. Therefore it has been assumed that users performed exercises at a stage when they have regained their muscle strength. On the other hand, the structural configuration of the elbow exoskeleton is fixed at assistive mode during exercise where users are allowed to exhibit their joint movement as per their strength. Joint parameters during exercises are measured by the physical sensor attached to the exoskeleton and Kinect sensor.

We have used Microsoft Kinect V2 which has been improved from its previous version Xbox 360 for capturing joint position and depth data. It is now integrated with inbuilt Unity plugin software. Therefore, users played the game in Unity platform with the help of Kinect sensor and the game was programmed in Microsoft visual studio. Kinect V2 is available in the market and its price is affordable for common people. Therefore users can perform exercises in home environment.

User's health condition was determined based on the measured joint parameters. User's performance score was computed based on its joint angle and time to reach. If users are able to score high value, it shows better recovery. In order to boost their effort, the game was supported by motivating voice commands which were fed after crossing a set level. To evaluate the system, two healthy subjects participated in the game. A comparative study has been undertaken and the data collected from the physical sensor on the elbow exoskeleton and the Kinect measurement was used to evaluate the measurement accuracy. Considering the exoskeleton data as reference, the average measurement errors in joint angle are within $1 \%$ for subject 1 and $0.44 \%$ for subject 2 . The errors in torque measurement are within $5.55 \%$ and $3.58 \%$ respectively. The length of arm segments measured by Kinect and standard biomechanics rule are also computed. The above results showed that rehabilitation parameters can be monitored accurately using Kinect sensor. This contactless measurement technique will give a pathway to use this technique for controlling the rehabilitation parameters in future. It can also be used solo for guiding rehabilitation exercise at advanced stages of recovery without using an exoskeleton. It will create a contactless and self-motivating system.

\section{REFERENCES}

[1] Piron, L., Turolla, A., Agostini, M., Zucconi, C., Tonin, P., Piccione, F., and Dam, M., 2009, "Assessment and treatment of the upper limb by means of virtual reality in post-stroke patients," Stud Health Technol Inform, 145, pp. 55-62.

[2] Smith, G. V., Silver, K. H., Goldberg, A. P., and Macko, R. F., 1999, "“Task-oriented" exercise improves hamstring strength and spastic reflexes in chronic stroke patients," Stroke, 30(10), pp. 2112-2118.

[3] Smithard, D., 2017, "Stroke in frail older people," Geriatrics, 2(3), p. 24.

[4] Shin, J.-H., Ryu, H., and Jang, S. H., 2014, "A task-specific interactive game-based virtual reality rehabilitation system for patients with stroke: a usability test and two clinical experiments," Journal of Neuroengineering and Rehabilitation, 11(1), p. 32.

[5] Tsekleves, E., Paraskevopoulos, I. T., Warland, A., and Kilbride, C., 2016, "Development and preliminary evaluation of a novel low cost VR-based upper limb stroke rehabilitation platform using Wii technology," Disability and Rehabilitation: Assistive Technology, 11(5), pp. 413-422.

[6] Levin, M. F., Weiss, P. L., and Keshner, E. A., 2015, "Emergence of virtual reality as a tool for upper limb rehabilitation: incorporation of motor control and motor learning principles," Physical therapy, 95(3), pp. 415-425.

[7] Goude, D., Björk, S., and Rydmark, M., "Game design in virtual reality systems for stroke rehabilitation," Proc. MMVR, pp. 146-148.

[8] Roy, A. K., Soni, Y., and Dubey, S., "Enhancing effectiveness of motor rehabilitation using kinect motion sensing technology," Proc. 2013 Global Humanitarian Technology Conference: South Asia Satellite (GHTC-SAS), IEEE, pp. 298-304. 
[9] Egglestone, S. R., Axelrod, L., Nind, T., Turk, R., Wilkinson, A., Burridge, J., Fitzpatrick, G., Mawson, S., Robertson, Z., and Hughes, A. M., "A design framework for a home-based stroke rehabilitation system: Identifying the key components," Proc. 2009 3rd International Conference on Pervasive Computing Technologies for Healthcare, IEEE, pp. $1-8$.

[10] Windolf, M., Götzen, N., and Morlock, M., 2008, "Systematic accuracy and precision analysis of video motion capturing systems-exemplified on the Vicon-460 system," Journal of Biomechanics, 41(12), pp. 2776-2780.

[11] Hillstrom, H. J., Garg, R., Kraszewski, A., Lenhoff, M., Carter, T., Backus, S. I., Wolff, A., Syrkin, G., Cheng, R., and Wolfe, S. W., 2014, "Development of an anatomical wrist joint coordinate system to quantify motion during functional tasks," Journal of Applied Biomechanics, 30(4), pp. 586-593.

[12] Da Gama, A., Fallavollita, P., Teichrieb, V., and Navab, N., 2015, "Motor rehabilitation using Kinect: a systematic review," Games for Health Journal, 4(2), pp. 123-135.

[13] Sookhanaphibarn, K., Phukongchai, W., Santad, T., and Choensawat, W., "Towards Bilateral Upper-Limb Rehabilitation after Stroke using Kinect Game," Proc. 2018 IEEE 7th Global Conference on Consumer Electronics (GCCE), IEEE, pp. 818819.

[14] Obdrzalek, S., Kurillo, G., Ofli, F., Bajcsy, R., Seto, E., Jimison, H., and Pavel, M., "Accuracy and robustness of Kinect pose estimation in the context of coaching of elderly population," Proc. 2012 Annual International Conference of the IEEE on Engineering in Medicine and Biology Society (EMBC), IEEE, pp. 1188-1193.

[15] Lin, C.-H., Sun, P.-Y., and Yu, F., "Space connection: a new 3D tele-immersion platform for web-based gesturecollaborative games and services," Proc. Proceedings of the Fourth International Workshop on Games and Software Engineering, IEEE Press, pp. 22-28.

[16] Lugrin, J.-L., Latt, J., and Latoschik, M. E., "Avatar anthropomorphism and illusion of body ownership in VR," Proc. 2015 Virtual Reality (VR), IEEE, pp. 229-230.

[17] Antón, D., Goñi, A., Illarramendi, A., Torres-Unda, J. J., and Seco, J., "KiReS: A Kinect-based telerehabilitation system," Proc. 2013 IEEE 15th International Conference on eHealth Networking, Applications \& Services (Healthcom), IEEE, pp. 444-448.

[18] Jaume-i-Capó, A., Martínez-Bueso, P., Moyà-Alcover, B., and Varona, J., 2014, "Improving vision-based motor rehabilitation interactive systems for users with disabilities using mirror feedback," The Scientific World Journal, 2014.

[19] Chang, C.-Y., Lange, B., Zhang, M., Koenig, S., Requejo, P., Somboon, N., Sawchuk, A. A., and Rizzo, A. A., "Towards pervasive physical rehabilitation using Microsoft Kinect," Proc. PervasiveHealth, pp. 159-162.

[20] Guneysu, A., Siyli, R. D., and Salah, A. A., "Autoevaluation of motion imitation in a child-robot imitation game for upper arm rehabilitation," Proc. 2014 23rd IEEE International Symposium on Robot and Human Interactive Communication, IEEE, pp. 199-204.
[21] Ma, M., Proffitt, R., and Skubic, M., "Quantitative assessment and validation of a stroke rehabilitation game," Proc. 2017 IEEE/ACM International Conference on Connected Health: Applications, Systems and Engineering Technologies (CHASE), IEEE, pp. 255-257.

[22] Wang, X., Li, X., Wang, J., and Luo, T., "A Kinect-Based Trajectory Capturing and Tracking Scheme for Upper-Limb Robot-Aided Rehabilitation," Proc. 2017 IEEE 7th Annual International Conference on CYBER Technology in Automation, Control, and Intelligent Systems (CYBER), IEEE, pp. 249-254.

[23] Bouteraa, Y., Abdallah, I. B., and Elmogy, A. M., 2019, "Training of Hand Rehabilitation Using Low Cost Exoskeleton and Vision-Based Game Interface," Journal of Intelligent \& Robotic Systems, pp. 1-17.

[24] Regazzoni, D., Vitali, A., Rizzi, C., and Colombo, G., 2018, "A Method to Analyse Generic Human Motion With Low-Cost Mocap Technologies," (51739), p. V01BT02A009.

[25] Wochatz, M., Tilgner, N., Mueller, S., Rabe, S., Eichler, S., John, M., Voller, H., and Mayer, F., 2019, "Reliability and validity of the Kinect V2 for the assessment of lower extremity rehabilitation exercises," Gait \& Posture, 70, pp. 330-335.

[26] Manna, S. K., and Dubey, V. N., "Design Proposal for a Portable Elbow Exoskeleton," Proc. 2018 Design of Medical Devices Conference, American Society of Mechanical Engineers, pp. V001T003A013-V001T003A013.

[27] Winter, D. A., 1990, "Biomechanics and motor control of human motion," New York: Wiley-Interscience.

[28] Manna, S. K., and Dubey, V. N., "Design of a Game-based Rehabilitation System using Kinect Sensor," Proc. 2019 Design of Medical Devices Conference, American Society of Mechanical Engineers. 